\title{
卤代芳烃的无溶剂微波法高效合成有机锡化合物及原位自身偶联反应
}

\author{
潘春娇 ${ }^{a}$ 刘 敏 ${ }^{b}$ 段新红 ${ }^{*, c}$ \\ ( ${ }^{a}$ 北京林业大学材料科学与技术学院 北京 100083) \\ $\left({ }^{b}\right.$ 北京林业大学林学院 北京 100083$)$ \\ ( ${ }^{c}$ 北京林业大学理学院 北京 100083)
}

\begin{abstract}
摘要 采用无溶剂微波加热合成方法, 通过 $\mathrm{Pd}\left(\mathrm{PPh}_{3}\right)_{4}$ 催化卤代芳烃与 $\mathrm{Sn}_{2} \mathrm{Bu}_{6}$ 的反应, 成功地合成了一系列有机锡化合 物. 这一新方法不仅操作简单、反应快和效率高，而且副产物少、污染小. 同时，此方法还可顺利实现卤代芳烃的原位 自身偶联反应.
\end{abstract}

关键词 微波加热; 无溶剂; 有机锡化合物; 自身偶联; 原位

\section{Highly Efficient Synthesis of Stannanes from Aryl halides and Their in-situ Homo-Coupling under Microwave-Irradiated and Solvent-Free Conditions}

\author{
Pan, Chunjiao ${ }^{a} \quad{\text { Liu, } \text { Min }^{b} \quad \text { Duan, Xinhong }}^{*, b}$ \\ ( ${ }^{a}$ Material Science and Technology, Beijing Forestry University, Beijing 100083) \\ ( ${ }^{a}$ College of Forestry, Beijing Forestry University, Beijing 100083) \\ ( ${ }^{b}$ College of Science, Beijing Forestry University, Beijing 100083)
}

\begin{abstract}
A novel approach for the synthesis of a variety of aryl stannanes from aryl halides and $\mathrm{Sn}_{2} \mathrm{Bu}_{6}$ by using $\mathrm{Pd}\left(\mathrm{PPh}_{3}\right)_{4}$ as catalyst is developed under microwave-irradiated and solvent-free conditions. This method offers the advantages of not only being simple, rapid and highly efficient, but also producing little byproduct and reducing pollution resulting from the use of solvent. Also, the procedure is applicable to the in-situ homo-coupling of aryl halides.
\end{abstract}

Keywords microwave heating; solvent-free; stannanes; homo-coupling; in-situ

有机锡化合物 $\left(\mathrm{R}^{1} \mathrm{SnR}_{3}\right)$ 是有机合成中常用的金属有 机试剂 ${ }^{[1]}$. 正因为具有易制备、稳定性好、对水和空气 不敏感，且碳-锡键易断裂产生新化合物等突出优势， 使有机锡化合物广泛地应用于多种有机合成中. 其中, 它与卤代烃偶联所实施的 Stille 反应, 是构建碳一碳键最 为成功和有效的方法之一 ${ }^{[2]}$. 另一方面, 将 $\mathrm{R}^{1} \mathrm{SnR}_{3}$ 转化 为 $R^{1} I^{123} 、 R^{1} I^{125}$ 或 $R^{1} F^{18}$ 的反应也是制备放射性核素药 物的理想方法 ${ }^{[3]}$.

一般而言, 有机锡化合物即可通过有机锂或锌试剂 $\left(\mathrm{R}^{1} \mathrm{Li}\right.$ or $\left.\mathrm{R}^{1} \mathrm{Zn}\right)$ 以及格氏试剂 $\left(\mathrm{R}^{1} \mathrm{MgX}\right)$ 的金属转化反应制 备 ${ }^{[4]}$, 也可通过六烷基二锡 $\left(\mathrm{Sn}_{2} \mathrm{R}_{6}\right)$ 直接锡化卤代烷烃 $\left(\mathrm{R}^{1} \mathrm{X}\right)$ 来制备 ${ }^{[5]}$. 然而就金属转化反应而言, 当底物携有 活性基团(例如活泼氢)时往往会限制这一方法的使用. 因此, 利用卤代烷烃与 $\mathrm{Sn}_{2} \mathrm{R}_{6}$ 的反应制备有机锡化合物
就尤为重要. 这一方法的常规制备是: Pd 作催化剂, 卤 代烷烃 (一般是澳代或碘代烷烃)与 $\mathrm{Sn}_{2} \mathrm{R}_{6}$ 在如甲苯、 $N, N$ 二甲基甲酰胺( $\mathrm{DMF}$ 或三乙胺 $\left(\mathrm{Et}_{3} \mathrm{~N}\right)$ 等溶剂中回流而产 生有机锡化合物. 尽管这种制备方法行之有效, 但同时 也存在着溶剂量大、反应(回流)时间长、副反应多以及 产率低等缺陷. 尤其是结构复杂的生物活性分子(卤代 芳烃)的锡化反应，若采用此常规方法来实现，产率往 往都很低 ${ }^{[6]}$. 针对这一方法所存在的种种缺陷, 亟需将 其进行有效地改进，从而达到条件温和、操作简单、快 速而高效地制备有机锡化合物.

微波加热技术是近年来发展起来的一种新型绿色 化学方法, 目前已经广泛应用于化学合成领域 ${ }^{[7]}$. 与常 规加热方式相比, 利用微波加热进行化学反应的优势如 下: 1)大大提高反应速率和产率; 2)具有立体或区域选择

*E-mail: xinhong116@163.com

Received August 22, 2014; revised October 9, 2014; published online November 3, 2014. 
性；3)减少副产物和溶剂使用量；4)可进行无溶剂反应; 5)完成常规加热方式下不能进行的反应; 6)简化和提高 传统的合成方法等.

最近, 我们采用微波加热法成功地制备了一系列芳 香族三丁基锡化合物 ${ }^{[8]}$. 这一方法是以水为单一溶剂条 件下实施的, 必需强碱(如 $\mathrm{KOH}$ ) 和相转移催化剂作为添 加剂, 对水或强碱较为敏感的底物有一定的局限性. 而 在进一步改进该方法的过程中我们发现：当以 $\mathrm{DMF} / \mathrm{Et}_{3} \mathrm{~N}$ 混合液为添加剂时, 此反应不仅可在微波加 热和无溶剂的条件下顺利进行, 而且较常规方法大大缩 短了反应时间、大幅度提高了反应效率(图 1). 正因为无 溶剂反应和微波加热是绿色合成化学中最为重要的两 个组成部分, 将二者有机结合即无溶剂微波加热法, 是 目前有机领域中广泛关注的新合成方法 ${ }^{[9]}$.

本文利用无溶剂微波加热法在优化条件下制备多 种芳烃和杂芳烃的三丁基锡化合物，同时也通过这一方 法有效地进行了 Stille 自身偶联反应(图 1).

\section{1 结果与讨论}

\section{1 优化反应条件}

首先, 我们以 4-溴苯甲醚的锡化反应为模板探讨反 应温度、反应时间、 $\mathrm{Sn}_{2} \mathrm{Bu}_{6}$ 和 $\mathrm{Pd}$ 催化剂的用量以及添 加剂的种类/用量分别对产物 2a 产率的影响, 实验结果

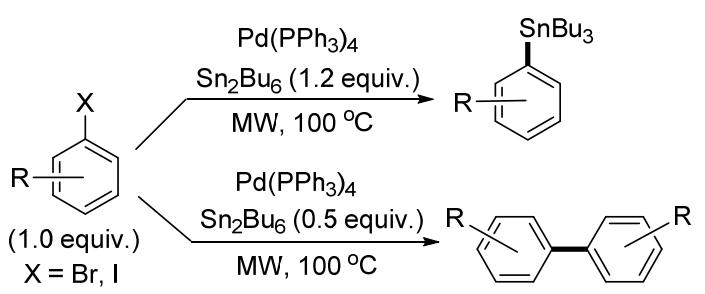

图 1 制备三丁基锡化合物和自身偶联产物

Figue 1 Synthesis of stannanes and homo-coupling products

如表 1 所示. 对于该反应所需的 Pd 催化剂而言, 实际上 $\mathrm{Pd}\left(\mathrm{PPh}_{3}\right)_{4}$ 最为经济而又具有较高的催化性能, 因此我 们选择它为本项研究的催化剂. 同时, 我们使用微波 $(300 \mathrm{~W})$ 加热该反应. 从表 1 的数据可知, 无溶剂条件下 若没有添加剂, 即使加热到 $100{ }^{\circ} \mathrm{C}$ 达 $6 \mathrm{~min}$, 该模板也 不发生任何化学反应(Entry 1). 以 $\mathrm{DMF}$ 或 $\mathrm{Et}_{3} \mathrm{~N}$ 作为单 一的添加剂时，提高反应温度或增加反应时间，反应的 结果都不理想(Entries $2 \sim 9$ ). 当 $\mathrm{DMF} / \mathrm{Et}_{3} \mathrm{~N}$ 等物质的量 (1/1)混合时, 该反应的产率提高到 61\% (Entry 10). 在此 基础上，我们继续探索 $\mathrm{DMF} / \mathrm{Et}_{3} \mathrm{~N}$ 的最佳配比. 当采用 $\mathrm{DMF} / \mathrm{Et}_{3} \mathrm{~N}=0.8 / 0.4$ (物质的量比)时, 在 $6 \mathrm{~min}$ 内 $2 \mathrm{a}$ 的产 率可达 92\% (Entry 11), 但继续延长反应时间或提高反 应温度，该反应的产率都没有明显的变化(Entries 12, 13). 接着, 我们考察了催化剂和 $\mathrm{Sn}_{2} \mathrm{Bu}_{6}$ 的用量对该反 应的影响(Entries 14 17). 实验结果表明: $\mathrm{Pd}\left(\mathrm{PPh}_{3}\right)_{4}$ 的

表 1 优化反应条件 ${ }^{a}$

Table 1 Optimization of the reaction conditions

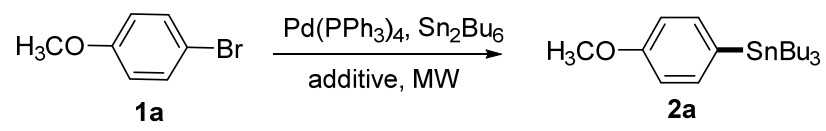

\begin{tabular}{|c|c|c|c|c|c|c|}
\hline Entry & $\mathrm{Sn}_{2} \mathrm{Bu}_{6}$ /equiv. & $\mathrm{Pd}\left(\mathrm{PPh}_{3}\right)_{4} / \mathrm{mol} \%$ & Temperature $/{ }^{\circ} \mathrm{C}$ & Time/min & Additive (equiv.) & Yield ${ }^{b} / \%$ \\
\hline 1 & 2.0 & 4 & 100 & 6 & None & None \\
\hline 2 & 2.0 & 4 & 100 & 6 & DMF (1.0) & Trace \\
\hline 3 & 2.0 & 4 & 100 & 6 & DMF (2.0) & 12 \\
\hline 4 & 2.0 & 4 & 100 & 6 & DMF (10.0) & 43 \\
\hline 5 & 2.0 & 4 & 120 & 8 & DMF (10.0) & 47 \\
\hline 6 & 2.0 & 4 & 80 & 6 & $\mathrm{Et}_{3} \mathrm{~N}(1.0)$ & None \\
\hline 7 & 2.0 & 4 & 80 & 6 & $\mathrm{Et}_{3} \mathrm{~N}(2.0)$ & Trace \\
\hline 8 & 2.0 & 4 & 80 & 6 & $\mathrm{Et}_{3} \mathrm{~N}(10.0)$ & 11 \\
\hline 9 & 2.0 & 4 & 80 & 8 & $\mathrm{Et}_{3} \mathrm{~N}(10.0)$ & 15 \\
\hline 10 & 2.0 & 4 & 100 & 6 & $\mathrm{DMF} / \mathrm{Et}_{3} \mathrm{~N}(1.0 / 1.0)$ & 61 \\
\hline 11 & 2.0 & 4 & 100 & 6 & $\mathrm{DMF} / \mathrm{Et}_{3} \mathrm{~N}(0.8 / 0.4)$ & 92 \\
\hline 12 & 2.0 & 4 & 120 & 6 & $\mathrm{DMF} / \mathrm{Et}_{3} \mathrm{~N}(0.8 / 0.4)$ & 87 \\
\hline 13 & 2.0 & 4 & 100 & 8 & $\mathrm{DMF} / \mathrm{Et}_{3} \mathrm{~N}(0.8 / 0.4)$ & 89 \\
\hline 14 & 2.0 & 6 & 100 & 6 & $\mathrm{DMF} / \mathrm{Et}_{3} \mathrm{~N}(0.8 / 0.4)$ & 93 \\
\hline 15 & 3.0 & 4 & 100 & 6 & $\mathrm{DMF} / \mathrm{Et}_{3} \mathrm{~N}(0.8 / 0.4)$ & 92 \\
\hline 16 & 1.2 & 4 & 100 & 6 & DMF/Et ${ }_{3} N(0.8 / 0.4)$ & 90 \\
\hline 17 & 1.0 & 4 & 100 & 6 & $\mathrm{DMF} / \mathrm{Et}_{3} \mathrm{~N}(0.8 / 0.4)$ & 88 \\
\hline $18^{c}$ & 2.0 & 5 & Reflux & 240 & Toluene (solvent) & $40(34)^{d}$ \\
\hline
\end{tabular}

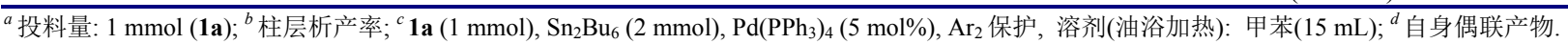


用量为 $4 \mathrm{~mol} \%, \mathrm{Sn}_{2} \mathrm{Bu}_{6}$ 的用量为 1.2 个物质的量时, 该 反应的产率可达 90\% (Entry 16). 同时我们也发现, 在 此最佳反应条件下，该反应并没有自身偶联的副产物生 成. 与之相比, 若采用常规的反应条件 ${ }^{[10]}$, 这一反应却 不可避免地产生了大量的自身偶联产物, 从而造成了产 率显著降低 $(40 \%$, Entry 18$)$.

\section{2 底物的拓展}

为了调查这一新方法的普适性, 我们进行了底物的 拓展实验, 结果见表 2. 在上述的最优化条件下, 我们 发现此方法适合多种类型的卤代芳烃和杂芳烃作为底 物, 而且官能团对反应的产率并没有显著的影响. 从表 2 中可以看出: 无论是缺电子的吡啶还是富电子的噻吩 体系，该反应都能顺利地进行(产率分别为 $83 \%$ 和 $86 \%$, Entries 1,2). 其中, 结构较为复杂 (如包含多个 $\mathrm{N}$ 或 $\mathrm{O}$ 杂 原子) 的生物活性分子 ${ }^{[11]}$, 使用这一方法也实现了高效 锡化, 反应结果较为理想(Entries 3 6). 同时我们发现, 即使底物中包含了活泼氢 $(\mathrm{N}-\mathrm{H})$ 也不影响该反应的成 功实施(产率为 $81 \%$, Entry 3). Entry 5 的实验结果表明: 就碘代芳烃而言, 即使连有强拉电子基团仍能被快速地 锡化并获得较高产率 $(88 \%)$ ). 另外, 对反应条件要求较 苛刻的官能团例如: $\mathrm{COOCH}_{3}$ (酯基，在碱性条件下易水 解), 在此反应条件下并没有发生明显的水解反应，而 且得到较高的产率 $(79 \%$, Entry 6$)$.

\section{3 自身偶联反应}

通常, Stille 自身偶联反应是指: 经纯化(或直接购 买)的有机锡化合物 $\left(\mathrm{RSnBu}_{3}\right)$ 与卤代烃 $(\mathrm{RX})$ 在 $\mathrm{Pd}$ 催化下 产生偶联, 从而形成自身偶联产物 ${ }^{[12]}$. 为了拓展无溶剂 微波加热法的应用范围，我们继续探索这一方法在 Stille 自身偶联反应中的应用. 由于该方法能高效、快速 地制备有机锡化合物，为实施步骤经济的原位 Stille 偶 联反应(一锅法, 消除了隔离/纯化有机锡化合物步骤) 提 供了可能.

的确，通过无溶剂微波加热法仅用 $4 \mathrm{~mol} \%$ $\mathrm{Pd}\left(\mathrm{PPh}_{3}\right)_{4}$ 就能连续地催化锡化反应和 Stille 反应，从而 实现自身偶联，结果见表 3. 一方面，溴代和碘代芳烃 都能顺利地发生 Stille 自身偶联反应; 另一方面, 无论 是富电子的卤代苯或噻吩体系还是缺电子的卤代杂芳 烃如吡啶或苯并噻唑体系都能很好地进行原位 Stille 反 应，得到目标偶联产物 3a～3d(产率 81 91\%, Entries $1 \sim 4)$. 此外，即使是连有酯基 $\left(\mathrm{COOC}_{2} \mathrm{H}_{5}\right)$ 的卤代芳烃同 样能实施这一反应，并得到了较高的产率 $(84 \%$, Entries $5)$.

\section{2 结论}

本文采用无溶剂微波加热法 $\left(100{ }^{\circ} \mathrm{C}\right)$, 在 $\mathrm{DMF} /$ $\mathrm{Et}_{3} \mathrm{~N}$ 为添加剂的条件下, 通过 $\mathrm{Pd}\left(\mathrm{PPh}_{3}\right)_{4}$ 催化卤代芳烃 或杂芳烃与 $\mathrm{Sn}_{2} \mathrm{Bu}_{6}$ 的直接锡化反应，成功地合成了一

表 2 底物的拓展 ${ }^{a}$

Table 2 Scope of the synthesis of stannanes

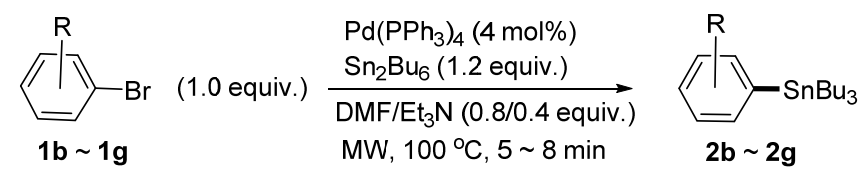

\begin{tabular}{|c|c|c|c|c|c|c|c|c|c|}
\hline Entry & Substrate 1 & Time/min & 2 & Yield $^{b} / \%$ & Entry & Substrate 1 & Time/min & 2 & Yield $^{b} / \%$ \\
\hline 1 & $1 b$ & 6 & $2 \mathrm{~b}$ & 83 & 4 & $1 e$ & 7 & $2 e$ & 91 \\
\hline 2 & 1c & 5 & $2 c$ & 86 & 5 & & 8 & $2 f$ & 88 \\
\hline 3 & & 8 & 2d & 81 & 6 & & 6 & $2 g$ & 79 \\
\hline
\end{tabular}

投料量: $1 \mathrm{mmol}(\mathbf{1 b} \sim \mathbf{1 g}) ;{ }^{b}$ 柱层析产率. 
表 3 无溶剂微波加热法的原位自身偶联反应 ${ }^{a}$

Table 3 The in-situ homo-coupling reaction under microwave-irradiated and solvent-free conditions

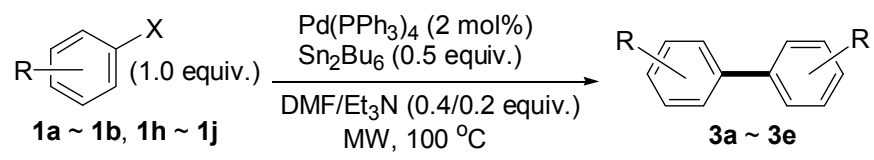

Entry Substrate $\mathbf{1}$

投料量: $2 \mathrm{mmol}(\mathbf{1} \mathbf{a} \sim \mathbf{1 b}, \mathbf{1 h} \sim \mathbf{1 j}){ }^{b}{ }^{\text {重结晶产率. }}$

系列具有高产率的有机锡化合物. 同时, 利用此方法顺 利地实现了原位自身偶联反应. 这一方法不仅具有操作 简单、反应快和效率高等突出优势，而且副产物少、污 染小, 为有机锡化合物的高效合成以及自身偶联化合物 的顺利制备提供了一条新的途径.

\section{3 实验部分}

\section{1 仪器与试剂}

熔点采用 RD-II 型显微熔点仪测定(未校正); 核磁 共振谱用 Bruker Avance DPX 400 (400 MHz)型核磁共 振仪测定, 氝代 $\mathrm{CHCl}_{3}$ 或 DMSO 作溶剂(TMS 作内标); 质谱用 TRACE 测定; 紫外-可见吸收光谱用 WFH-204B 型手提式紫外分析仪测定; TLC 板用 GF254(青岛海洋化 工有限公司)高效薄层层析硅胶板. 实验所用试剂均购 买于 Aldrich Chemicals 或 J \& K Scientific Ltd; 微波炉用 北京祥鹄科技发展有限公司生产的 XH-200A 微波合成 仪 (功率: $0 \sim 1000 \mathrm{~W}$; 温度: $0 \sim 260{ }^{\circ} \mathrm{C}$ ).

\section{2 实验方法}

\subsection{1 有机锡化合物的制备}

卤代物(1.0 mmol), $\mathrm{Sn}_{2} \mathrm{Bu}_{6}(0.7 \mathrm{~g}, 1.2 \mathrm{mmol})$ 以及 $\mathrm{Pd}\left(\mathrm{Ph}_{3} \mathrm{P}\right)_{4}(46 \mathrm{mg}, 40 \mu \mathrm{mol})$ 于反应器中混合, 加入 $\mathrm{DMF}$ $(58 \mathrm{mg}, 0.8 \mathrm{mmol}) / \mathrm{Et}_{3} \mathrm{~N}(40 \mathrm{mg}, 0.4 \mathrm{mmol})$ 的混合液. 微 波 $(300 \mathrm{~W}) 100{ }^{\circ} \mathrm{C}$ 加热, 待反应完毕后( TLC 跟踪), 反应
混合物经柱层析分离得到目标产物.

\section{2 .2 自身偶联反应}

将卤代物(2.0 mmol), $\mathrm{Sn}_{2} \mathrm{Bu}_{6}(0.6 \mathrm{~g}, 1.0 \mathrm{mmol})$ 以及 $\mathrm{Pd}\left(\mathrm{Ph}_{3} \mathrm{P}\right)_{4}(46 \mathrm{mg}, 40 \mu \mathrm{mol})$ 在反应器中混合, 加入 $\mathrm{DMF}$ (58 mg, $0.8 \mathrm{mmol}) / \mathrm{Et}_{3} \mathrm{~N}$ (40 mg, $\left.0.4 \mathrm{mmol}\right)$. 微波(300W) $100{ }^{\circ} \mathrm{C}$ 加热, 待反应完毕后(TLC 跟踪), 反应混合物经 重结晶分离得到目标产物.

\section{2 .3 化合物表征}

4-甲氧基苯基三丁基锡(2a) ${ }^{[13]}$ : 柱色谱纯化(流动 相: 环己烷), 无色粘稠油状物. ${ }^{1} \mathrm{H}$ NMR (400 MHz, $\left.\mathrm{CDCl}_{3}\right) \delta: 0.88(\mathrm{t}, J=14.6 \mathrm{~Hz}, 9 \mathrm{H}), 1.02$ (t, $J=16.3 \mathrm{~Hz}$, $6 \mathrm{H}), 1.30 \sim 1.35(\mathrm{~m}, 6 \mathrm{H}), 1.49 \sim 1.57(\mathrm{~m}, 6 \mathrm{H}), 3.79(\mathrm{~s}$, $3 \mathrm{H}), 6.90$ (d, $J=8.4 \mathrm{~Hz}, 2 \mathrm{H}), 7.37$ (d, $J=8.4 \mathrm{~Hz}, 2 \mathrm{H})$.

2-甲氧基-5-三丁基锡基吡啶 $(2 \mathbf{b})^{[14]}$ : 柱色谱纯化 [流动相: $V$ (乙酸乙酯 $) / V($ 环己烷 $)=1 / 10$ ], 无色粘稠油状 物. ${ }^{1} \mathrm{H}$ NMR (400 MHz, $\left.\mathrm{CDCl}_{3}\right) \delta: 0.88(\mathrm{t}, J=14.6 \mathrm{~Hz}$, $9 \mathrm{H}), 1.05$ (t, $J=16.2 \mathrm{~Hz}, 6 \mathrm{H}), 1.28 \sim 1.37(\mathrm{~m}, 6 \mathrm{H}), 1.49 \sim$ $1.57(\mathrm{~m}, 6 \mathrm{H}), 3.93(\mathrm{~s}, 3 \mathrm{H}), 6.74(\mathrm{~d}, J=8.0 \mathrm{~Hz}, 1 \mathrm{H}), 7.61$ (d, $J=8.1 \mathrm{~Hz}, 1 \mathrm{H}), 8.15$ (s, 1H).

2-噻吩基三丁基锡(2c) ${ }^{[15]}$ : 柱色谱纯化(流动相: 环 己烷), 无色粘稠油状物. ${ }^{1} \mathrm{H}$ NMR (400 $\left.\mathrm{MHz}, \mathrm{CDCl}_{3}\right) \delta$ : $0.89(\mathrm{t}, J=14.6 \mathrm{~Hz}, 9 \mathrm{H}), 1.10(\mathrm{t}, J=16.2 \mathrm{~Hz}, 6 \mathrm{H}), 1.31 \sim$ $1.36(\mathrm{~m}, 6 \mathrm{H}), 1.55 \sim 1.59(\mathrm{~m}, 6 \mathrm{H}), 7.20(\mathrm{~d}, J=3.0 \mathrm{~Hz}$, 
1H), 7.27 (t, $J=4.2 \mathrm{~Hz}, 1 \mathrm{H}), 7.65$ (d, $J=4.6 \mathrm{~Hz}, 1 \mathrm{H})$.

2-(4-溴苯基)-6-甲基喹唑啉-4(3H)-酮(1d ${ }^{[16]}$ : 将 2-

氨基-5-甲基苯甲酰胺 $(0.15 \mathrm{~g}, 1 \mathrm{mmol})$ 与 3 -溴苯甲醛 (0.24 g, $1.3 \mathrm{mmol})$ 加入反应器, 滴入 $3 \sim 5$ 滴 DMF 使体 系完全混匀. 微波加热 $\left(300 \mathrm{~W}, 110{ }^{\circ} \mathrm{C}\right) 12 \mathrm{~min}$. 待体系 冷却后有固体析出, 甲醇重结晶得白色晶体. 产率 74\%. m.p. $141 \sim 142{ }^{\circ} \mathrm{C} ;{ }^{1} \mathrm{H}$ NMR (400 MHz, DMSO) $\delta: 2.46$ (s, 3H), $7.66(\mathrm{t}, J=10.7 \mathrm{~Hz}, 2 \mathrm{H}), 7.75$ (d, $J=10.7 \mathrm{~Hz}, 2 \mathrm{H})$, $7.95(\mathrm{~s}, 1 \mathrm{H}), 8.11(\mathrm{~d}, J=10.7 \mathrm{~Hz}, 2 \mathrm{H})$. Anal. calcd for $\mathrm{C}_{15} \mathrm{H}_{11} \mathrm{BrN}_{2} \mathrm{O}$ : C 57.16, H 3.52, N 8.99; found C 57.18, H $3.14, \mathrm{~N} 9.03$.

2-(4-三丁基锡基苯基)-6-甲基喹唑啉-4(3H)-酮(2d): 柱色谱纯化 [流动相: $V$ (乙酸乙酯 $) / V($ 环己烷 $)=1 / 5$ ]得到 黄色针状晶体. m.p. $31 \sim 32{ }^{\circ} \mathrm{C}$; ${ }^{1} \mathrm{H}$ NMR $(400 \mathrm{MHz}$, $\left.\mathrm{CDCl}_{3}\right) \delta: 0.90(\mathrm{t}, J=14.6 \mathrm{~Hz}, 9 \mathrm{H}), 1.17$ (t, $J=16.2 \mathrm{~Hz}$, $6 \mathrm{H}), 1.34 \sim 1.39(\mathrm{~m}, 6 \mathrm{H}), 1.57 \sim 1.61(\mathrm{~m}, 6 \mathrm{H}), 2.46(\mathrm{~s}$, $3 \mathrm{H}), 7.36$ (d, $J=10.1 \mathrm{~Hz}, 2 \mathrm{H}), 7.77$ (d, $J=10.0 \mathrm{~Hz}, 1 \mathrm{H})$, 7.89 (d, $J=10.0 \mathrm{~Hz}, 1 \mathrm{H}), 8.16$ (d, $J=10.2 \mathrm{~Hz}, 2 \mathrm{H}), 8.44$ $(\mathrm{s}, 1 \mathrm{H}), 11.51(\mathrm{~s}, \mathrm{NH}, 1 \mathrm{H}) ;{ }^{13} \mathrm{C} \mathrm{NMR}\left(100 \mathrm{MHz}, \mathrm{CDCl}_{3}\right) \delta$ : $9.83,13.70,17.53,27.38,29.10,120.06,126.83,127.34$, $129.71,130.14,134.27,141.47,142.05,142.62,149.44$, 151.64, 164.02; MS (ESI) $m / z(\%): 524.1\left([\mathrm{M}-\mathrm{H}]^{-}, 100\right)$.

2-[3,4-(亚甲二氧基)苯基]-6-溴苯并噻唑 $(\mathbf{1 e})^{[17]}$ : 将 2-氨基-5-溴苯硫酚( $0.41 \mathrm{~g}, 2 \mathrm{mmol})$ 与胡椒醛 $(0.30 \mathrm{~g}, 2$ $\mathrm{mmol}$ )加入反应器, 用少量乙醇溶解使其混合均匀, 旋 转蒸发掉乙醇, 微波加热 $\left(300 \mathrm{~W}, 120{ }^{\circ} \mathrm{C}\right)$ 混合物 $6 \mathrm{~min}$. 待体系冷却后, 乙醇重结晶得淡黄色颗粒状晶体. 产率 77\%. m.p. 173 $174{ }^{\circ} \mathrm{C} ;{ }^{1} \mathrm{H}$ NMR (400 MHz, $\left.\mathrm{CDCl}_{3}\right) \delta$ : $6.07(\mathrm{~s}, 2 \mathrm{H}), 6.90(\mathrm{~d}, J=10.9 \mathrm{~Hz}, 1 \mathrm{H}), 7.56 \sim 7.59(\mathrm{~m}$, 3H), 7.61 (d, $J=10.9 \mathrm{~Hz}, 1 \mathrm{H}), 7.87$ (d, $J=10.9 \mathrm{~Hz}, 1 \mathrm{H})$, $8.00(\mathrm{~s}, 1 \mathrm{H})$. Anal. calcd for $\mathrm{C}_{14} \mathrm{H}_{8} \mathrm{BrNO}_{2} \mathrm{~S}$ : C 50.32, $\mathrm{H}$ 2.41, N 4.19; found C 50.28, H 2.34, N 4.04.

2-[3,4-(亚甲二氧基)苯基]-6-三丁基锡基苯并噻唑 (2e): 柱色谱纯化 [流动相: $V$ (乙酸乙酯 $) / V$ (环己烷 $)=$ 1/20], 黄色粘稠油状物. 产率 $89 \%$. ${ }^{1} \mathrm{H}$ NMR (400 MHz, $\left.\mathrm{CDCl}_{3}\right) \delta: 0.90(\mathrm{t}, J=14.6 \mathrm{~Hz}, 9 \mathrm{H}), 1.11(\mathrm{t}, J=16.3 \mathrm{~Hz}$, $6 \mathrm{H}), 1.32 \sim 1.38(\mathrm{~m}, 6 \mathrm{H}), 1.53 \sim 1.61(\mathrm{~m}, 6 \mathrm{H}), 6.05(\mathrm{~s}$, 2H), 6.89 (d, $J=10.0 \mathrm{~Hz}, 1 \mathrm{H}), 7.53$ (d, $J=10.0 \mathrm{~Hz}, 1 \mathrm{H})$, $7.60(\mathrm{~d}, J=9.9 \mathrm{~Hz}, 2 \mathrm{H}), 7.95(\mathrm{~s}, 1 \mathrm{H}) \delta: 7.97$ (d, $J=10.0$ $\mathrm{Hz}, 1 \mathrm{H}) ;{ }^{13} \mathrm{C} \mathrm{NMR}\left(100 \mathrm{MHz}, \mathrm{CDCl}_{3}\right) \delta: 9.86,13.69$, $27.39,29.10,101.70,107.57,108.64,122.32,122.48$, $128.21,128.97,133.76,135.06,138.82,148.35,150.02$, 153.94, 167.02; MS (ESI) $m / z(\%): 544.3\left([\mathrm{M}+2 \mathrm{H}]^{+}\right.$, 100).

$N$-(4-三丁基锡基苯基)邻苯二甲酰亚胺(2f): 柱色谱
纯化 [流动相: $V$ (乙酸乙酯 $) / V($ 环己烷 $)=1 / 5]$, 无色晶体. m.p. $20 \sim 21{ }^{\circ} \mathrm{C} ;{ }^{1} \mathrm{H}$ NMR $\left(400 \mathrm{MHz}, \mathrm{CDCl}_{3}\right) \delta: 0.90$ (t, $J=14.7 \mathrm{~Hz}, 9 \mathrm{H}), 1.08(\mathrm{t}, J=16.3 \mathrm{~Hz}, 6 \mathrm{H}), 1.32 \sim 1.37(\mathrm{~m}$, $6 \mathrm{H}), 1.52 \sim 1.60(\mathrm{~m}, 6 \mathrm{H}), 7.38(\mathrm{~d}, J=10.0 \mathrm{~Hz}, 2 \mathrm{H}), 7.60$ $(\mathrm{d}, J=9.9 \mathrm{~Hz}, 2 \mathrm{H}), 7.78$ (d, $J=10.6 \mathrm{~Hz}, 2 \mathrm{H}), 7.95$ (d, $J=$ $10.6 \mathrm{~Hz}, 2 \mathrm{H}) ;{ }^{13} \mathrm{C} \mathrm{NMR}\left(100 \mathrm{MHz}, \mathrm{CDCl}_{3}\right)$ : 9.68, 13.69, 27.42, 29.08, 123.74, 125.73, 131.42, 131.84, 134.36, 137.08, 142.66, 167.37; MS (ESI) $m / z(\%): 536.1$ ([M+ $\left.\mathrm{Na}+\mathrm{H}]^{+}, 60\right), 291(100)$.

3-甲基-2-苯基-6-三丁基锡基-4-苯并吡喃酮-8-甲酸 甲酯 $(\mathbf{2 g})$ : 柱色谱纯化 [流动相: $V$ (乙酸乙酯) $/ V$ (环己 烷 $)=1 / 5$ ], 淡黄色油状物. ${ }^{1} \mathrm{H}$ NMR $\left(400 \mathrm{MHz}, \mathrm{CDCl}_{3}\right) \delta$ : $0.90(\mathrm{t}, J=14.6 \mathrm{~Hz}, 9 \mathrm{H}), 1.15(\mathrm{t}, J=16.2 \mathrm{~Hz}, 6 \mathrm{H}), 1.30 \sim$ $1.39(\mathrm{~m}, 6 \mathrm{H}), 1.52 \sim 1.60(\mathrm{~m}, 6 \mathrm{H}), 2.26(\mathrm{~s}, 3 \mathrm{H}), 3.96(\mathrm{~s}$, $3 \mathrm{H}), 7.53(\mathrm{dt}, J=5.4,4.8 \mathrm{~Hz}, 3 \mathrm{H}), 7.81(\mathrm{~d}, J=9.6 \mathrm{~Hz}$, $2 \mathrm{H}), 8.30(\mathrm{~s}, 1 \mathrm{H}), 8.54(\mathrm{~s}, 1 \mathrm{H}) .{ }^{13} \mathrm{C} \mathrm{NMR}(100 \mathrm{MHz}$, $\left.\mathrm{CDCl}_{3}\right) \delta: 9.9,11.86,13.66,27.29,29.01,52.40,117.91$, $119.53,122.34,128.43,129.34,130.42,133.22,138.23$, $138.71,143.37,154.69,160.72,165.51,178.58$; MS (ESI) $m / z(\%): 607.1\left([\mathrm{M}+\mathrm{Na}+\mathrm{H}]^{+}, 100\right)$.

$4,4^{\prime}$-二甲氧基联苯 $(3 a)^{[18]}$ : 甲醇重结晶, 白色晶体. m.p. $177 \sim 178{ }^{\circ} \mathrm{C} ;{ }^{1} \mathrm{H}$ NMR $\left(400 \mathrm{MHz}, \mathrm{CDCl}_{3}\right) \delta: 3.84$ (s, $6 \mathrm{H}), 6.96(\mathrm{dt}, J=3.0,5.0 \mathrm{~Hz}, 4 \mathrm{H}), 7.47((\mathrm{dt}, J=3.0,5.0$ $\mathrm{Hz}, 4 \mathrm{H})$.

2,2'-甲氧基-5,5'-联吡啶 $(3 \mathbf{b})^{[19]}$ : 乙醇重结晶, 白色 晶体. m.p. $105 \sim 106{ }^{\circ} \mathrm{C} ;{ }^{1} \mathrm{H}$ NMR $\left(400 \mathrm{MHz}, \mathrm{CDCl}_{3}\right) \delta$ : 4.02 (s, 6H), 6.86 (d, $J=8.6 \mathrm{~Hz}, 2 \mathrm{H}), 7.72(\mathrm{dd}, J=2.5,2.5$ $\mathrm{Hz}, 2 \mathrm{H}), 8.34$ (d, $J=2.3 \mathrm{~Hz}, 2 \mathrm{H})$.

$3,3^{\prime}$-联噻吩 $(\mathbf{3 c})^{[20]}$ : 乙醇重结晶, 白色晶体. m.p. $131 \sim 132{ }^{\circ} \mathrm{C} ;{ }^{1} \mathrm{H}$ NMR (400 MHz, $\left.\mathrm{CDCl}_{3}\right) \delta: 7.32 \sim 7.35$ (m, 4H), $7.36 \sim 7.38(\mathrm{~m}, 2 \mathrm{H})$.

$2,2^{\prime}$-联苯并噻唑 $(\mathbf{3 d})^{[21]}$ : 甲醇重结晶, 白色晶体, m.p. $>250{ }^{\circ} \mathrm{C} ;{ }^{1} \mathrm{H}$ NMR (400 MHz, $\left.\mathrm{CDCl}_{3}\right) \delta: 7.04$ (dt, $J=0.9,8.2 \mathrm{~Hz}, 2 \mathrm{H}), 7.28(\mathrm{tt}, J=8.3,3.9 \mathrm{~Hz}, 2 \mathrm{H}), 7.58$ (dd, $J=0.5,8.2 \mathrm{~Hz}, 4 \mathrm{H})$.

联苯-4,4'-二甲酸二乙酯 $(3 e)^{[22]}$ : 乙醇重结晶, 白色 晶体. m.p. $109 \sim 110{ }^{\circ} \mathrm{C} ;{ }^{1} \mathrm{H}$ NMR $\left(400 \mathrm{MHz} \mathrm{CDCl}_{3}\right) \delta$ : 1.38 (t, $J=14.3 \mathrm{~Hz}, 6 \mathrm{H}), 4.35$ (q, $J=21.3 \mathrm{~Hz}, 4 \mathrm{H}), 6.89$ (d, $J=8.7 \mathrm{~Hz}, 4 \mathrm{H}), 7.95(\mathrm{~d}, J=8.6 \mathrm{~Hz}, 4 \mathrm{H})$.

辅助材料(Supporting Information) 化合物 1d、1e 和 $\mathbf{2 a} \sim 2 \mathbf{g}$ 的 ${ }^{1} \mathrm{H} N M R 、{ }^{13} \mathrm{C}$ NMR 图谱以及 $\mathbf{3 a} \sim \mathbf{3 e}$ 的 ${ }^{1} \mathrm{H}$ NMR 图谱. 这些材料可以免费从本刊网站(http://siocjournal.cn/)上下载. 


\section{References}

[1] (a) Lu, A. L.; Wang, F. J.; Huang, D. F.; Wang, K. H.; Su, Y. P.; Xu, Y. L.; Hu, Y. L. Chin. J. Org. Chem. 2014, 34, 948 (in Chinese).

(陆爱玲, 王风娇, 黄丹凤, 王克虎, 苏瀛鹏, 徐艳丽, 胡雨来, 有机化学, 2014, 34, 948.)

(b) Zhang, X. Y.; Song, H. B.; Tang, L. F. Acta Chim. Sinica 2011, 69, 2567 (in Chinese).

(张晓燕, 宋海滨, 唐良富, 化学学报, 2011, 69, 2567.)

(c) Zhang, Z. H.; Xu, W. J.; Lu, Y. B.; Xiong, Y. Q.; An, D. L.; Peng, Z. H. Acta Chim. Sinica 2007, 65, 2905 (in Chinese)

(张正华, 徐伟箭, 卢彦兵, 熊远钦, 安德烈, 彭志鸿, 化学学报, 2007, 65, 2905.)

(d) Song, X. Q.; Zhong, G. Y.; Huang, Y. Q.; Xie, Q. L. Chin. J. Org. Chem. 2002, 22, 735 (in Chinese).

(宋雪清, 钟桂云, 黄艳琴, 谢庆兰, 有机化学, 2002, 22, 735.)

[2] (a) Zeng, J.; Liu, K. M.; Duan, X. F. Org. Lett. 2013, 15, 5342.

(b) Cho, S. H. Chem. Soc. Rev. 2011, 40, 5068

(c) Li, W. Y.; Zhao, D. M.; Xiong, X. Q.; Ma, Q. Q.; Cheng, M. S. Chin. J. Org. Chem. 2011, 31, 784 (in Chinese).

(李文燕, 赵冬梅, 熊绪琼, 马倩倩, 程卯生, 有机化学, 2011, 31, 784.)

(d) Wang, D. P.; Zhang, X. D.; Liang, Y.; Li, J. H. Chin. J. Org. Chem. 2006, 26, 19 (in Chinese).

(王德平, 张旭东, 梁云, 李金恒, 有机化学, 2006, 26, 19.)

(e) Nicolaou, K. C. Angew. Chem., Int. Ed. 2005, 44, 4442.

(f) Yu, Z. K.; Wang, S. H. Chin. J. Org. Chem. 1993, 13, 579 (in Chinese).

(余正坤, 王世华, 有机化学, 1993, 13, 579.)

(g) Wang, Z. Y.; Guo, X. B. Chin. J. Org. Chem. 1989, 9, 124 (in Chinese).

(王昭显, 郭秀斌, 有机化学, 1989, 9, 124.)

[3] (a) Ono, M. Chem. Pharm. Bull. 2009, 57, 1029.

(b) Qu, W. C. J. Med.Chem. 2007, 50, 3380.

(c) Zhuang, Z. P. J. Med. Chem. 2001, 44, 1905.

[4] (a) Hayashi, T.; Ishigedani, M. Tetrahedron 2001, 57, 2589.

(b) Knochel, P.; Singer, R. D. Chem. Rev. 1993, 93, 2117.

(c) Iddon, B.; Lim, B. L. J. Chem. Soc., Perkin Trans. 1 1983, 271.

(d) Gilman, H.; Rosenberg, S. D. J. Am. Chem. Soc. 1953, 75, 2507.

[5] (a) Handy, C. J.; Manoso, A. S.; McElroy, W. T.; Seganish, W. M.; DeShong, P. Tetrahedron 2005, 61, 12201.

(b) Zhu, X.; Blough, B. E.; Caroll, F. I. Tetrahedron Lett. 2000, 41, 9219.

(c) Sandosham, J.; Undheim, K. Acta Chem. Scand. 1989, 43, 684.

(d) Azizian, H.; Eaborn, C.; Pidcock, A. J. Organomet. Chem. 1981, 215, 49 .

[6] (a) Duan, X. H.; Qiao, J. P.; Yang, Y.; Cui, M. C.; Zhou, J. N.; Liu, B. L. Bioorg. Med. Chem. 2010; 18: 1337.

(b) Suzuki, M.; Doi, H.; Kato, K.; BjoÈrkman, M.; LaÊngstroÈm, B.; Watanabe, Y.; Noyori, R. Tetrahedron 2000, 56, 8263.

[7] (a) Li, X. L.; Wang, W.; Li, R.; Zhang, P. Z.; Chen, H. Chin. J. Org. Chem. 2012, 32, 1519 (in Chinese).

(李小六, 王玮, 李锐, 张平竹, 陈华, 有机化学, 2012, 32, 1519.)

(b) Kappe, C. O. Chem. Soc. Rev. 2008, 37, 1127.

(c) Dallinger, D. Chem. Rev. 2007, 107, 2563.

(d) Roberts, B. A. Acc. Chem. Res. 2005, 38, 653.

(e) Melucci, M.; Barbarella, G.; Sotgiu, G. J. Org. Chem. 2002, 67, 8877.

(f) Lu, M. W.; Hu, W. X. Chin. J. Org. Chem. 1995, 15, 561 (in Chinese).

(陆模文, 胡文祥, 有机化学, 1995, 15, 561.)
[8] Tan, X.; Zhou, Z. J.; Zhang, J. X.; Duan, X. H. Eur. J. Org. Chem. 2014, 5153 .

[9] (a) Shi, Z. C.; Zhao, Z. G.; Li, H.; Tan, J. Chin. J. Org. Chem. 2014, 34, 572 (in Chinese).

(石治川, 赵志刚, 李晖, 谭昫, 有机化学, 2014, 34, 572.)

(b) Lai, Q. Y.; Liao, R. S.; Wu, S. Y.; Zhang, J. X.; Duan, X. H. New J. Chem. 2013, 37, 4069.

(c) Moseley, J. D.; Kappe, C. O. Green Chem. 2011, 13, 794.

(d) Lu, J.; Ge, H. G.; Bai, Y. J. Chin. J. Org. Chem. 2002, 22, 782 (in Chinese)

(路军，葛红光，白银娟，有机化学， 2002，22，782.)

(e) Varma, R. S. Green Chem. 1999, 1, 43.

[10] (a) Wang, B.; Qin, L.; Neumann, K. D.; Uppaluri, S.; Cerny, R. L.; DiMagno, S. G. Org. Lett. 2010, 12, 3352.

(b) Mase, N.; Nakai, Y.; Ohara, N.; Yoda, H.; Takabe, K.; Tanaka, F.; Barbas III, C. F. J. Am. Chem. Soc. 2006, 128, 734.

(c) Narayan, S.; Muldoon, J.; Finn, M. G.; Fokin, V. V.; Kolb, H. C.; Sharpless, K. B. Angew. Chem., Int. Ed. 2005, 44, 3275.

[11] (a) Rubio, S.; León, F.; Quintana, J.; Cutler, S.; Estévez, F. Eur. J. Med. Chem. 2012, 55, 284.

(b) Hayakawa, M.; Kaizawa, H.; Moritomo, H.; Koizumi, T.; Ohishi, T.; Okada, M.; Ohta, M.; Tsukamoto, S.; Parker, P.; Workman, P.; Waterfield, M. Bioorg. Med. Chem. 2006, 14, 6847.

(c) Shinji, C.; Nakamura, T.; Maeda, S.; Yoshida, M.; Hashimoto, Y.; Miyachi, H. Bioorg. Med. Chem. Lett. 2005, 15, 4427.

(d) Mathis, C. A.; Wang, Y. M.; Holt, D. P.; Huang, G. F.; Debnath, M. L.; Klunk, W. E. J. Med. Chem. 2003, 46, 2740.

[12] (a) Tsvelikhovsky, D.; Blum, J. Eur. J. Org. Chem. 2008, 2417.

(b) Li, J.-H.; Liang, Y.; Wang, D.-P.; Liu, W.-J.; Xie, Y.-X.; Yin, D.-L. J. Org. Chem. 2005, 70, 2832.

[13] Tang, P. P.; Furuya, T.; Ritter, T. J. Am. Chem. Soc. 2010, 132, 12150 .

[14] Wei, J. F.; Jiao, J.; Feng, J. J.; Lv, J.; Zhang, X. R.; Shi, X. Y.; Chen, Z. G. J. Org. Chem. 2009, 74, 6283.

[15] Pinault, T.; Chérioux, F.; Therrien, B.; Süss-Fink, G. Heteroat. Chem. 2004, 15, 121.

[16] The compound can also be synthesized by the other method, see: (a) Wang, M.; Zhang, T. T.; Song, Z. G. Chin. J. Org. Chem. 2010, 30, 740 (in Chinese).

(王敏，张婷婷，宋志国，有机化学, 2010, 30, 740.)

(b) Hayakawa, M.; Kaizawa, H.; Moritomo, H.; Koizumi, T.; Ohishi, T.; Okada, M.; Ohta, M.; Tsukamoto, S.; Parker, P.; Workman, P.; Waterfield, M. Bioorg. Med. Chem. 2006, 14, 6847.

[17] The compound can also be synthesized by the other method, see: (a) Ke, F.; Wu, W.; Lin, C.; Li, P. Chin. J. Org. Chem. 2013, 33, 2559 (in Chinese).

(柯方, 吴雯, 林晨, 李鹏, 有机化学, 2013, 33, 2559.)

(b) Mathis, C. A.; Wang, Y. M.; Holt, D. P.; Huang, G. F.; Debnath, M. L.; Klunk, W. E. J. Med. Chem. 2003, 46, 2740.

[18] Lee, P. H.; Seomoon, D.; Lee, K. Org. Lett. 2005, 7, 343.

[19] (a) Qian, Y. L.; Wang, C.; Tao, X. C.; Huang, J. L. Chin. J. Org. Chem. 2003, 23, 1264 (in Chinese)

(钱延龙, 王晨, 陶晓春, 黄吉玲, 有机化学, 2003, 23, 1264.)

(b) Parry, P. R.; Wang, C. S.; Batsanov, A. S.; Bryce, M. R.; Tarbit, B. J. Org. Chem. 2002, 67, 7541.

[20] Billingsley, K.; Buchwald, S. L. J. Am. Chem. Soc. 2007, 129, 3358.

[21] Rai, C.; Braunwarth, J. B. J. Org. Chem. 1961, 26, 3434.

[22] Cahiez, G.; Moyeux, A.; Buendia, J.; Duplais, C. J. Am. Chem. Soc. 2007, 129, 13788.

(Li, L.; Lu, Z.) 